\title{
Factores asociados al bajo peso al nacer en un hospital de Huánuco
}

\author{
Associated factors of low birth \\ weight, in Carlos Showing \\ Ferrari hospital in Huánuco, \\ Perú.
}

\section{Henry Lowell Allpas-Gómez, ${ }^{1}$ Jarvis Raraz-Vidal ${ }^{2}$ y Omar Raraz-Vidal ${ }^{2}$}

\section{RESUMEN}

INTRODUCCIÓN. El bajo peso al nacer (BPN) es un importante indicador de salud fetal, neonatal y de las condiciones de la gestación. Овлетіvo: identificar factores asociados al BPN en el Hospital Carlos Showing Ferrari de Huánuco, Perú. Material y método. Estudio descriptivo, retrospectivo de corte transversal. Se realizó en el servicio de Ginecoobstetricia del Hospital Carlos Showing Ferrari de Huánuco. Participantes. Madre e hijos atendidos en el servicio de Gineco-obstetricia. InTERVEncIones. Según los criterios de inclusión y exclusión se obtuvo, 801 madreshijos registrados en el Sistema Informático Perinatal (SIP) del 2011. Se utilizó un instrumento de recolección validado, se obtuvo información del SIP e historias clínicas, durante 6 meses. Se realizó el análisis descriptivo; para el análisis inferencial se usó la prueba ji cuadrado con una significancia del $95 \%$. Se empleó el paquete estadístico SPSS y Microsoft Excel. Resultados. De los 801 nacidos, el peso promedio fue $3217,8( \pm 446,1)$ gramos $y$ una prevalencia de BPN de 6,2\%. Los factores asociados que resultaron estadisticamente significativos fueron: la primiparidad con $p=0,013$ (IC-95\%: 1,16-3,81); número de controles prenatales (menor de cuatro) con $p<0,05$ (IC95\%: 2,63-8,74) y parto pretérmino con $p<0,05$ (IC95\%: $0,01-0,25)$. Conclusión. La prevalencia del bajo peso al nacer en el Hospital Carlos Showing Ferrari de Huánuco fue 6,2 \%. La primiparidad, el inadecuado control prenatal y el parto pretérmino fueron los factores asociados.

Palabras clave. Recién nacido, recién nacido de bajo peso, factores de riesgo, trabajo de parto prematuro.

\section{SUMMARY}

INTRODUCTION. Low birth weight (LBW) is an important indicator of fetal, neonatal health and the conditions of pregnancy. Овлестіve. To identify the associated factors of low birth weight, in Carlos Showing Ferrari Hospital in HuanuCO. Materials AND methods. Retrospective, descriptive cross-sectional study. It was performed in the GynecologyObstetrics service of Carlos Showing Ferrari Hospital, Huanuco. Participants. Mothers and children treated in the Gynecology-Obstetrics Service. Interventions: According to inclusion and exclusion criteria, it obtained 801 mothers-children, registered in the Perinatal Information System (SIP) of 2011. One validated instrument was used for collection, the SIP information and medical history were obtained for six months. Descriptive analysis was conducted, for the inferential analysis chi-square test was used with a significance of $95 \%$. SPSS and Microsoft Excel were used. REsults. Of 801 births, the average weight was $3217,8( \pm 446,1)$ grams and a prevalence of LBW 6,2\%. Associated factors that were statistically significant were: primiparity with $p=0,013$ (C195\%: 1,16-3,81), number of prenatal visits (<4) with $p<0,05(C 195 \%: 2,63-8,74)$ and preterm birth with $p<0,05$ (C195\%: 0,01-0,25). ConCLusION. The prevalence of low birth weight in Carlos Showing Ferrari Hospital, Huanuco was 6,2\%. First childbirth, inadequate antenatal control and preterm birth were main correlated factors for low birth weight in our study.

KEY WORDS. Infant, newborn, infant, low birth weight risk factors, obstetric labor, premature. 


\section{INTRODUCCIÓN}

El bajo peso al nacer (BPN) ha sido definido por la Organización Mundial de Salud (OMS) como el peso del neonato menor de 2500 gramos, durante la primera hora de vida. ${ }^{\mathrm{I}}$ El BPN tiene una probabilidad de morir 20 veces myor que un recién nacido con peso mayor. ${ }^{1,2}$

La reducción del BPN constituye una importante contribución en las metas de Desarrollo del Milenio para disminuir la morbimortalidad infantil. Según la Unicef, la incidencia mundial de BPN fue de $14 \%^{3}$ y en América Latina y el Caribe, de 5,3\% a $15 \%{ }^{4}$ En Perú, fue 6,9\%, un valor comparable con los de Costa Rica, Argentina y República Dominicana. ${ }^{4}$

Diversos estudios describen la asociación del BPN con diversos trastornos durante el primer año de vida, entre los que destacan el retraso de desarrollo neurológico, ${ }^{5}$ la hemorragia cerebral, alteraciones respiratorias, el riesgo de presentar asfixia al momento de su nacimiento y otras enfermedades que obligan a una hospitalización prolongada. ${ }^{6}$

Diversas condiciones maternas durante el embarazo, entre ellas la terapia antirretroviral, raza negra, ${ }^{7}$ anemia, infección de la vía urinaria, preeclampsia, rotura prematura de membranas, ganancia de peso ponderal materna menor de $8 \mathrm{~kg}$, el síndrome hipertensivo del embarazo, ${ }^{8}$ factores psicosociales $^{9}$ y fumadoras, $^{10}$ son un riesgo predictor de BPN. Otros factores asociados son la hemoglobina fetal glicosilada elevada ${ }^{11}$ y la prematuridad. ${ }^{12}$

En el Hospital Carlos Showing Ferrari (HCSF), del distrito de Amarilis, región Huánuco, se atiende a una población con bajos niveles socioeconómicos provenientes de zonas urbanomarginales y rurales, cuyas familias en mayor cuantía carecen de servicios básicos. El objetivo del estudio fue determinar los posibles factores asociados con el BPN en el HCSF.

\section{MATERIAL Y MÉTODO}

Estudio descriptivo, retrospectivo, observacional de corte transversal en el período de un año, de enero a diciembre de 2011, realizado en el servicio de Ginecoobstetricia del HCSF en el área urbana del distrito de Amarilis, región de Huánuco, Perú.
La población del estudio fue la población total que asistió a ser atendida en el servicio de Ginecoobstetricia del HCSF. En el período de estudio fue de 826 pacientes, de los cuales 801 cumplieron con los criterios de selección.

Los criterios de selección tomados fueron los siguientes: de inclusión, nacido en el HCSF, en el año 2011; de exclusión, nacidos en otras dependencias de salud o en su domicilio y los natimuertos.

El instrumento de recolección de datos utilizado fue el Sistema Informático Perinatal (SIP), desarrollado por el Centro Latino Americano de Perinatología/ Salud de la Mujer y Reproductiva (CLAP/SMR) para vigilancia epidemiológica de la salud materna y perinatal de América Latina desde 1983.

El registro de la información se realizó a partir de la Historia Clínica Perinatal base (HCP-b), documento existente en el lugar donde la gestante realiza su control prenatal (CPN), adicionando la información del trabajo de parto, del parto y del posparto inmediato, que duró seis meses.

Las variables analizadas fueron el BPN (peso $<2500 \mathrm{~g}$ ) y el sexo del recién nacido, la edad, el estado civil, el estado nutricional, la talla y el hábito de fumar de la madre, la paridad, el número de consultas prenatales y el diagnóstico de patología durante la gestación. La base de datos del SIP fue procesada en el programa estadístico SPSS y los datos se presentaron en tablas. Las variables continuas se analizaron a través de medidas de tendencia central y dispersión; las variables categóricas, como proporciones. Para la ilustración de los resultados se empleó el software Microsoft Excel. Para establecer la asociación entre cada una de las variables, se usaron las pruebas de ji cuadrado y de Fisher, con un intervalo de confianza del $95 \%$ (IC95\%), y fueron consideradas como significativos valores de $\mathrm{p}<0,0513$.

El estudio fue aprobado por un comité de ética del HCSF, se garantizó el anonimato a través de códigos por paciente.

\section{RESULTADOS}

En el período del 1 de enero al 31 de diciembre de 2006, de un total de 826 HCP-b, se analizaron 801 que cumplieron con los criterios de inclusión. Dentro de las características de las gestantes, se 
Tabla 1. Características generales de las gestantes en el Hospital Carlos Showing Ferrari de Huánuco, Perú

Característica de la gestante Frecuencia Porcentaje

$\triangle$ Edad

- Menor de 20 años

- De 20 a 34 años

- Mayor de 35 años

253

31,6

56,9

11,5

$\triangle$ Estado civil

- Soltera

182

22,7

- Casada

139

17,4

- Conviviente

474

59,2

- Otro

3

0,4

$\triangle$ Nivel educativo

- Analfabeta

39

4,9

- Primaria incompleta

- Primaria completa

- Secundaria incompleta

- Secundaria completa

- Superior

\section{$\triangle$ Paridad}

- Primíparas

- Secundíparas

- Multíparas

$\triangle$ Estado nutricional

- Desnutridas

- Normopesos

- Sobrepesos

- Obesas

28

$\triangle$ Control prenatal

- No adecuados

- Adecuados

$\triangle$ Talla materna

- Menor o igual a $150 \mathrm{~cm}$

- Mayor de $150 \mathrm{~cm}$

$\triangle$ Parto pretérmino

- Sin parto pretérmino

793
8

99

- Con parto pretérmino
Tabla 2. Bajo peso al nacer en el Hospital Carlos Showing Ferrari de Huánuco, Perú

\begin{tabular}{|c|c|c|}
\hline Peso al nacer & Frecuencia & Porcentaje \\
\hline$\triangle$ Bajo peso al nacer & 50 & 6,2 \\
\hline$\triangle$ Normopeso & 751 & 93,8 \\
\hline Total & 801 & 100,0 \\
\hline
\end{tabular}

nutricional adecuado, la talla mayor de $150 \mathrm{~cm}$ y el parto normal. Tabla 1.

De los datos analizados, el peso de los neonatos en el estudio varió de 1000 a $4700 \mathrm{~g}$, con una media de $3217,8 \mathrm{~g}$, una desviación estándar de 446,1 y siguió una distribución normal. Se observó que $53 \%$ de nacidos vivos fueron de sexo femenino y el $47 \%$, de sexo masculino.

El BPN se presentó en 50 casos, con una prevalencia de 6,2\%. Tabla 2.

El análisis estadístico de las variables muestra diferencias estadísticamente significativas en las variables control prenatal, paridad y parto pretérmino. Tabla 3.

Tabla 3. Análisis estadístico de las variables en relación con el bajo peso al nacer en el Hospital Carlos Showing Ferrari de Huánuco, Perú

Variable

P IC $95 \%$

$\triangle$ Control prenatal inadecuado

$<0,05 \quad 2,63-8,74$

$\triangle$ Presencia de parto prematuro

$<0,05 \quad 0,01-0,25$

$\triangle$ Madre primípara

$0,013 \quad 1,16-3,81$

$\Delta$ Edad materna $<20$ años

$0,075 \quad 1,01-3,37$

$\Delta$ Estado civil materno inestable $\quad 0,124 \quad 0,66-3,81$

$\Delta$ Talla materna baja menor de $150 \mathrm{~cm} \quad 0,208 \quad 0,82-3,22$

$\triangle$ Patología en el embarazo

$0,392 \quad 0,37-1,47$

$\Delta$ Sexo femenino del recién nacido $\quad 0,640 \quad 0,49-1,54$

$\triangle$ Madre analfabeta

$0,768 \quad 0,18-3,43$

$\Delta$ Presencia de desnutrición materna $0,801 \quad 0,40-3,27$

Fuente: Historia Clínica Perinatal Básica (HCPB), del Hospital Carlos Showing Ferrari de Huánuco, Perú. encontró mayor frecuencia en el grupo etário de 20 a 34 años, el estado civil conviviente, la instrucción secundaria completa, la primiparidad, el estado 


\section{DISCUSIÓN}

La Unicef y la OMS estimaron que la prevalencia de BPN para Perú disminuyó de $11 \%(2000)^{1}$ a $6,9 \%$ (2012). ${ }^{4}$ Según la región natural, en la sierra encontraron $10,2 \%{ }^{14}$ pero el INEI reportó $9,8 \% .^{15}$ Estudios peruanos mostraron una prevalencia de BPN de $10,2 \%$ en Sullana ${ }^{16}$ y $4,32 \%$ en Ica. ${ }^{17}$ La prevalencia de BPN en el presente estudio fue $6,2 \%$, semejante a la hallada en China $(6,1 \%),{ }^{8}$ Costa Rica $(7 \%)^{4}$ y México $(6 \%),{ }^{18}$ pero menor a la encontrada en México (8,5\%), ${ }^{4}$ Argentina $(10 \%)^{12}$ y Colombia $(8,98 \%) .^{19}$

En la población estudiada, se encontró asociación estadística entre el BPN y tres variables: control prenatal, primiparidad y parto prematuro. Los estudios anteriores muestran gran variedad de factores que dependen de la región estudiada. La variable inadecuado control prenatal es el factor asociado al BPN más frecuente, ${ }^{20}$ y concuerda con los hallazgos de diferentes autores como Ticona Rendón (Minsa) ${ }^{14}$ y Concha (Sullana) ${ }^{16}$ y de otros países como Cuba $^{21}$ y Colombia; ${ }^{20}$ mientras que discrepa con lo hallado por Santos Rivera en el Hospital Félix Torrealva Gutiérrez, EsSalud, Ica, ${ }^{17}$ que, además, cuenta con una población de nivel socioeconómico distinto a la del presente estudio.

La primiparidad es otro factor asociado a mayor riesgo de tener un hijo con BPN, que concuerda

\section{Agradecimientos}

Agradecemos al Hospital Carlos Showing Ferrari de Huánuco, los asesores y otros profesionales de la salud, en apoyo institucional, revisión del estudio y apoyo logístico.

\section{REFERENCIAS BIBLIOGRÁFICAS}

I. World Health Organization [sede Web-Internet]. The World Health Report 2005. Make every mother and child count. The greatest risks to life are in its beginning. Geneva:WHO; 2005:7981 . [accesado el 3 de mayo de 2014]. Disponible en: http://www. who.int/whr/2005/en/

2. Unicef [base de datos en Internet]. La desnutrición Infantil. "Causas, consecuencias y estrategias para su prevención y tratamiento". 20I I. España: Unicef; 201 I [accesado el 3 de mayo de 20/4]. Disponible en: http://www.unicef.es/sites/www.unicef. es/files/Dossierdesnutricion.pdf con los hallazgos en el hospital Nacional de Sullana ${ }^{16}$ y el estudio de Ticona Rendón (Minsa), ${ }^{14}$ pero difiere con los estudios de Santos Rivera ${ }^{17} y$ el de hospitales en China, ${ }^{8}$ lo que podría deberse a las diferencias socioeconómicas entre las dos poblaciones.

El parto pretérmino también fue un factor asociado con BPN en diversas instituciones como la clínica universitaria (Medellín, Colombia), ${ }_{1}^{20}$ policlínico (Cuba), ${ }^{22}$ de primer nivel de atención, Argentina, ${ }^{12}$ un hospital materno, ${ }^{8}$ hospitales de una provincia china (China). ${ }^{8}$ A veces, el parto prematuro fue la única variable asociada al BPN. ${ }^{20}$

El estudio fue limitado por el diseño retrospectivo que dependía de la recolección de datos de las HCP-b llenadas con anterioridad.

Los resultados encontrados en este estudio no deben extrapolarse a la población general porque las gestantes atendidas en el HCSF no representan a la región huanuqueña.

\section{CONCLUSIONES}

La prevalencia de bajo peso al nacer en el Hospital Carlos Showing Ferrari de Huánuco fue 6,2\% para el año 2011. El inadecuado control prenatal, el parto pretérmino y la primiparidad fueron los factores con asociación significativa al BPN. 
5. Goos TG, Dudink J, Reiss IK. Cerebral hypoxia immediately following delivery of very low birth weight infants.J Pediatr. 2014; 3476(I3): I494-1497.

6. Health policy council Sotsdoh. Early Child development. Tehran: Ministry of Health and Medical Education; 20II.

7. Slyker JA, Patterson J,Ambler T, Richardson BA, et al. Correlates and outcomes of preterm birth, low birth weight, and small for gestational age in HIV-exposed uninfected infants. BMC Pregnancy Childbirth. 20I4;I4(I):7-I2.

8. Chen Y, Li G, Ruan Y, Zou L,Wang X, ZhangW.An epidemiological survey on low birth weight infants in China and analysis of outcomes of full-term low birth weight infants. BMC Pregnancy and Childbirth. 20I3; I3:242-4.

9. González GJ, Vega MG. Factores de riesgo del peso al nacer desfavorable en áreas periféricas de Guadalajara, México. Cad. Saúde Públ. 1995; I (2):27|-280.

10. Jorge Vargas D, Soriano RE, Tejeda Matos SE, Vargas Vasquez E, Duran Agramente R, Valenzuela de los Santos ML, Lerebours Y. Perfil socio-demográfico de madres con hijos de bajo peso al nacer en el Hospital maternidad Nuestra Señora de la Altagracia. Rev Med Dom. 20I I;72(I):I37-I45.

I I. Pfab T, Slowinski T, Godes M, Halle H, Priem F, Hocher B. Low birth weight, a risk factor for cardiovascular diseases in later life, is already associated with elevated fetal glycosylated hemoglobin at birth. Circulation. 2006; I 14:1687-1692.

12. Couceiro M, Passamai M, Contreras N, Zimmer M, Cabianca G, Mayorga M, et al.Variables biológicas y sociales de embarazadas y peso al nacer de sus hijos, controladas por el primer nivel de atención (Salta,Argentina). Rev Antropo. 2009; 19:7-21.

13. Hernández Sampieri R, Fernández Collado C, Baptista Lucio MP. Metodología de la Investigación. 5. ${ }^{a}$ Ed. México D. F.: McGrawHill; 2010.

14. Ticona-Rendón M, Huanco-Apaza D,Ticona Vildoso M. Incidencia y factores de riesgo de bajo peso al nacer en poblacion atendida en hospitales del Ministerio de Salud del Perú. Ginecol Obstet Mex. 20I2;80(2):5I-60.

15. INEl. Indicadores de resultados de los programas estrategicos, 2010. Lima: INEl; 2010 [accesado el 3 de mayo de 2014] Disponible en: http://desa.inei.gob.pe/endes/Investigaciones/ Libro_Endes.pdf

16. Chávez W, Concha G. Bajo peso al nacer. Factores de riesgo departamento de Ginecología y Obstetricia y servicio de Neonatología, Hospital Apoyo III Sullana Minsa. Ginecol Obstet (Perú). 200I;47(I):47-52.

17. Rivera S,Vargas C, Quintanilla Y. Factores de riesgo de bajo peso al nacer en el Hospital Félix Torrealva Gutiérrez, EsSalud, Ica. Agosto 200 I - febrero 2002. Rev Per Epi. 2003; I I (I):56-63.

18. Naciones Unidas. Objetivos de desarrollo del Milenio. Comunicado de prensa 2010. México: Naciones Unidas; 2010 [accesado el 3 de mayo de 2014]. Disponible en: http://www. un.org/es/millenniumgoals/pdf/PR_Global_MDG09_SP.pdf

19. Profamilia. Encuesta Nacional de Demografía y Salud. Bogotá 2010. [Internet]. Colombia: ENDS; 2010 [accesado el 3 de mayo de 2014]. Disponible en: http://www.measuredhs.com/pubs/pdf/ FR246/FR246.pdf

20. Vélez MP, Barros F, Echavarría LG, Hormaza MP. Prevalencia de bajo peso al nacer y factores maternos asociados unidad de atención y protección materno infantil de la Clínica Universitaria Bolivariana, Medellín, Colombia. Rev Col Obs Ginecol. 2006;57: 264-270.

21. Cabrales Escobar JA, Sáenz Darías L, Grau Espinosa MA, et al. Factores de riesgo de bajo peso al nacer en un hospital cubano, 1997-2000. Rev Panam Salud Publica. 2002;12(3):180-184.

22. Rodríguez PL, Hernández J, Reyes A. Bajo peso al nacer.Algunos factores asociados a la madre Policlínico docente "José Jacinto Milanés" de Matanzas. Rev Cubana Obstet Ginecol. 2006;32(3). [accesado el 3 de mayo de 20I4]. Disponible en: http://bvs.sld.cu/ revistas/gin/vol32_3_06/gin0l306.htm

\section{Correspondencia}

Henry Allpas-Gómez

henryl.allpasg@gmail.com

\section{Conflictos de interés}

Los autores declaran no tener conflictos de interés durante el planteamiento, ejecución de la investigación y la elaboración del artículo para su publicación.

Fecha de recepción: 27 de marzo de 2014

Fecha de aceptación: 28 de abril de 2014 